\title{
Positron emission tomography for myocardial viability assessment before myocardial revascularization in a patient with extremely impaired left ventricular systolic function - advanced diagnosis and therapy in heart failure
}

\author{
Agata Krawczyk-Ożóg, Renata Rajtar-Salwa, Adam Gębka, Beata Bobrowska, Stanisław Bartuś, Dariusz Dudek \\ $2^{\text {nd }}$ Department of Cardiology, Institute of Cardiology, Jagiellonian University Medical College, Krakow, Poland
}

Adv Interv Cardiol 2017; 13, 2 (48): 173-175

DOI: https://doi.org/10.5114/pwki.2017.68051

In patients with heart failure caused by coronary artery disease (CAD), assessment of myocardial viability and successful revascularization seems to be crucial to improve the function of the left ventricle. Importantly, available data are inconclusive regarding the usefulness of myocardial viability tests for the decision concerning revascularization in patients with left ventricular (LV) dysfunction and CAD [1]. Multiple observational studies have reported that viability imaging will be instrumental in such patients. However, data from prospective studies failed to confirm that [2].

A 55-year-old man, a former smoker with a history of CAD and arterial hypertension, was admitted to our department to assess myocardial viability and the possibility of coronary revascularization. Two years before, he suffered from ST-segment elevation myocardial infarction of the antero-lateral wall successfully treated with percutaneous coronary intervention $(\mathrm{PCI})$ of the left anterior descending artery (LAD). Six months prior to admission the patient was hospitalized again at another ward due to unstable angina and underwent $\mathrm{PCl}$ of the right coronary artery. In the coronary angiogram ostial left circumflex artery $(C x)$ stenosis was found (Figure $1 \mathrm{~A}$ ). The patient reported symptoms of chronic heart failure (NYHA class III) and angina (CCS class II) with marked limitation of activities during normal exertion, with no symptoms at rest. The echocardiography showed severe systolic dysfunction of the LV with LV ejection fraction of $13 \%$, akinesis with thinning of the medial segment of lateral wall, akinesis of the septum, 2/3 distal of the anterior and posterior walls and significant hypokinesis of the inferior wall and basal segments of the posterior and anterior walls of the LV. No significant valvular disease was revealed. Dobutamine stress echocardiography (DSE) $(15 \mu \mathrm{g} / \mathrm{kg} / \mathrm{min})$ did not confirm the presence of contractile reserve of the LV. However, due to symptoms of angina the diagnostics were expanded by single-photon emission computed tomography (SPECT) and F18-fluorodeoxyglucose positron emission tomography (PET). The SPECT examination, using technetium-99m sestamibi, was performed. The level of the pharmaceutical agent uptake at rest in the lateral wall (more than $50 \%$ of maximal uptake) suggested preserved viability. The patient exercised on the treadmill to a maximum load of 7.9 MET. After exercise, the examination revealed evidence of a transient perfusion defect in the infero-lateral wall (worse perfusion in stress oblique/coronal slices 70-67 in row $C$ than in the corresponding rest slices 79-76 in row B). Additionally, PET imaging showed the presence of heterogeneous acquisition of the glucose radiotracer in the segments of the lateral wall (Figure 2). Transiency of SPECT perfusion defects and the heterogeneous acquisition might be considered as proof of myocardial viability and suggest a potential benefit from myocardial revascularization.

Due to extremely reduced LV ejection fraction and high risk of periprocedural complications, an LV assist device (HeartMate PHP, Thoratec Corporation, CA, USA) was used during $\mathrm{PCl}$. The procedure was performed by the femoral access. After the balloon predilation $(2.5 \times 20 \mathrm{~mm}$; $14 \mathrm{~atm})$ in LMCA and CX, implantation of a drug-eluting stent, Xience $3.0 / 23 \mathrm{~mm}$ (Abbott Vascular - Santa Clara, USA), up to 12-16 atm, was performed. Then, the proximal optimization technique with a non-compliant balloon $(3.5 \times 8 \mathrm{~mm}, 16 \mathrm{~atm})$ was applied. Finally, the kissing balloons technique was used (Figure $1 \mathrm{~B}$ ). No procedure-related complications were observed.

Corresponding author:

Agata Krawczyk-Ożóg MD, 2 ${ }^{\text {nd }}$ Department of Cardiology, Institute of Cardiology, Jagiellonian University Medical College, 17 Kopernika St, 31-501 Krakow, Poland, phone: +48 1242471 81, e-mail: krawczyk.ozog@gmail.com

Received: 12.10 .2016 , accepted: 11.01.2017. 

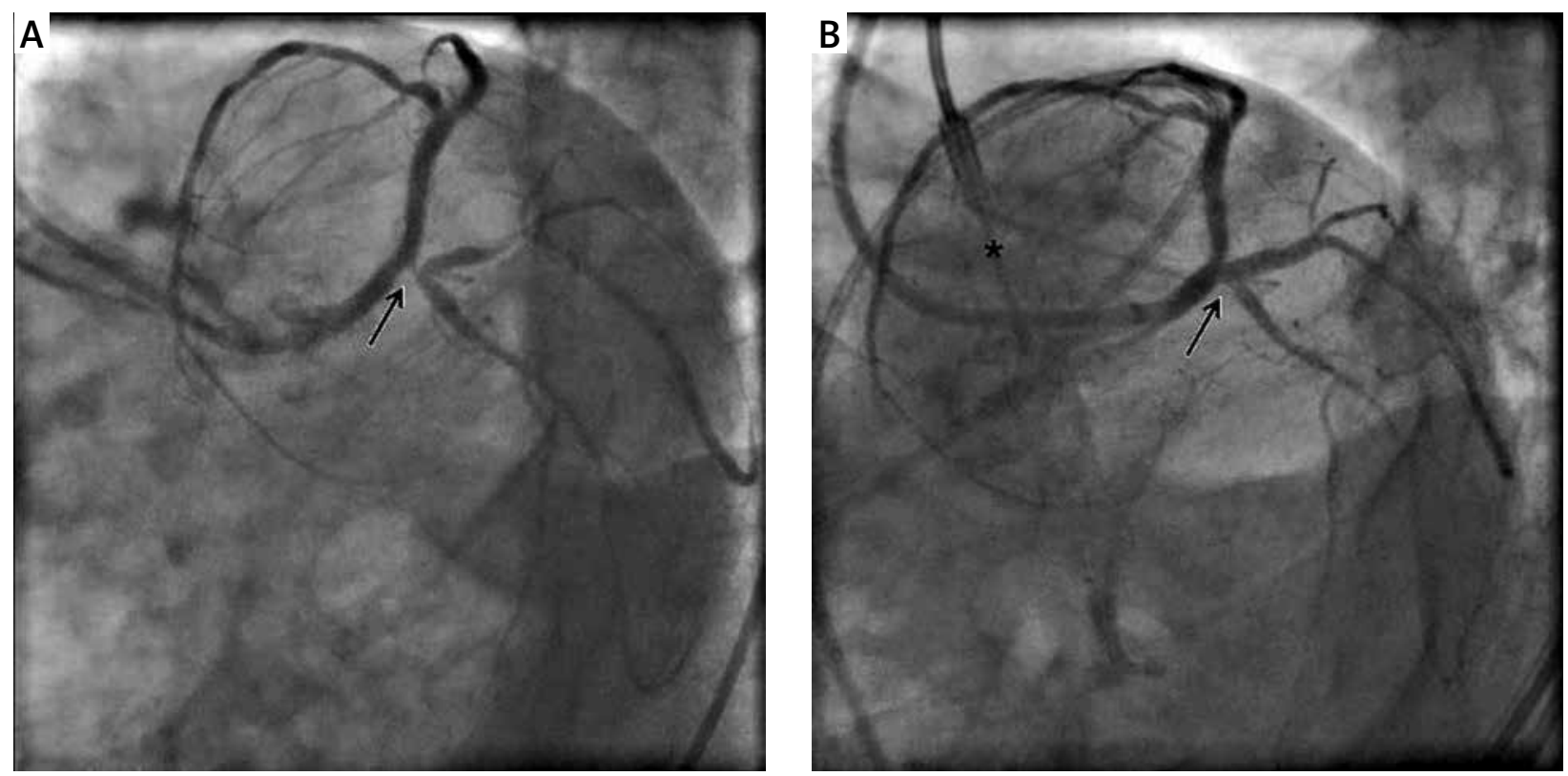

Figure 1. Ostial left circumflex artery stenosis before (A) and after (B) percutaneous coronary intervention with left ventricular assist device support. HeartMate PHP device marked with *; Stenosis and effect of interventions marked with arrows

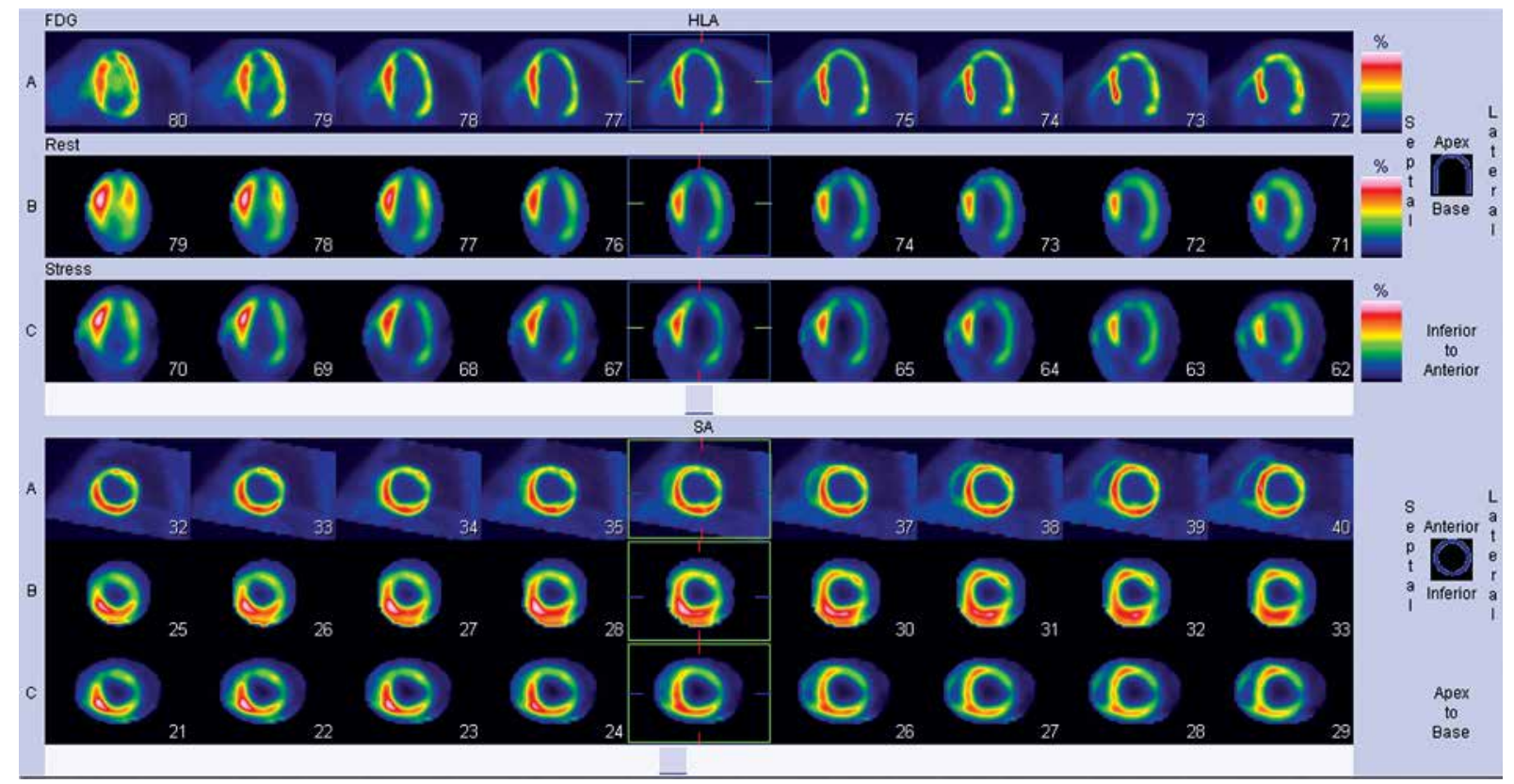

Figure 2. Assessment of myocardial viability by nuclear imaging techniques before intervention: A - F18-fluorodeoxyglucose positron emission tomography, B - single-photon emission computed tomography using technetium-99m sestamibi - Rest, C - single-photon emission computed tomography using technetium-99m sestamibi - Stress - after exercise treadmill test. The SPECT (B, C) and PET (A) showed that in the segments of the lateral wall acquisition of the radiotracer was present

The previous pharmacological treatment of heart failure was maintained. The patient had no indications for cardiac resynchronization therapy. After 1 year, the LV ejection fraction was increased up to $21 \%$. The most important effect was a significant reduction in the severity of symptoms and increased exercise tolerance (NYHA class I).
Akinesis of the walls of the LV is regarded as an irreversible condition and diagnostics of heart failure is not extended in such cases. In this particular case DSE was not sufficient to confirm myocardial viability, which however was confirmed by SPECT and PET imaging. The PET has higher sensitivity for the detection of viability 
but has lower specificity in comparison to DSE and SPECT $[3,4]$. The $\mathrm{PCl}$ with use of the LV assist device as periprocedural hemodynamic support may be a feasible and safe revascularization method in high-risk patients with severe heart failure or excessive comorbidities [5].

\section{Conflict of interest}

The authors declare no conflict of interest.

\section{References}

1. Orlandini A, Castellana N, Pascual A, et al. Myocardial viability for decision-making concerning revascularization in patients with left ventricular dysfunction and coronary artery disease: a meta-analysis of non-randomized and randomized studies. Int J Cardiol 2015; 182: 494-9.

2. Patel P, Ivanov A, Ramasubbu K. Myocardial viability and revascularization: current understanding and future directions. Curr Atheroscler Rep 2016; 18: 32.

3. Partington SL, Kwong RY, Dorbala S. Multimodality imaging in the assessment of myocardial viability. Heart Fail Rev 2011; 16: 381-95.

4. Rizzello V, Poldermans D, Bax JJ. Assessment of myocardial viability in chronic ischemic heart disease: current status. Q J Nucl Med Mol Imaging 2005; 49: 81-96.

5. Al-Rashid F, Hildebrandt HA, Kahlert P. Protected percutaneous coronary intervention - a new road for success in treating complex patients. Postep Kardiol Interw 2016; 12: 200-2. 